\title{
Letter to the Editor regarding: "Treatment patterns and survival outcomes in patients with cervical cancer complicated by complete uterine prolapse: a systematic review of literature"
}

\author{
Erin A. Brennand ${ }^{1} \cdot$ Robyn N. Banerjee ${ }^{2}$
}

Published online: 16 June 2016

(C) The International Urogynecological Association 2016

Sir,

We read with interest the review by Matsuo et al. of case reports regarding treatment of cervical cancer in the setting of procidentia [1]. We compliment the authors for highlighting a rare but complex clinical scenario. However, the data presented do not support their conclusion that, compared with primary radiation, "surgery-based treatment may have a positive effect on survival outcome in cervical cancer patients with complete uterine prolapse".

Such a conclusion would necessitate controlling for key patient and treatment variables including staging methodology, lymph node status, radiotherapy dose, target volume, and chemotherapy use. Tumor size is only reported in 16 of 78 patients. One-quarter of the patients treated with radiation alone received whole pelvic radiotherapy without brachytherapy. This is by definition palliative intent treatment and should not be compared with curative-intent therapies. Chemotherapy use is not controlled for, although concurrent chemoradiotherapy has been established as standard of care in patients with locally advanced cervical cancer since 2000 [2]. Finally, the authors' contention that "surgery-

Erin A. Brennand

erin.brennand@albertahealthservices.ca

1 Department of Obstetrics \& Gynecology, Division of Urogynecology, University of Calgary, North Tower, Foothills Medical Centre, 1403, 29 Street NW, Calgary, AB T2N 2T9, Canada

2 Department of Oncology, Division of Radiation Oncology, University of Calgary, Calgary, AB T2N 1N4, Canada based treatment was associated with significantly better disease-specific overall survival" cannot be supported given that the cause of death was "not stated" in the majority of patients (49 of 78).

Given the rarity of these coexisting conditions it is unsurprising that treatment approaches are heterogeneous. This very fact, however, undermines the ability to draw a robust conclusion about the relative merits of radiation versus surgical approaches in this scenario. Despite this, we would argue that because of the practical difficulties in delivering curative-intent radiotherapy in the setting of procidentia (due to challenges in target delineation, daily set-up reproducibility, and feasibility of brachytherapy treatment), surgery should be considered as the first-line approach in this setting.

Compliance with ethical standards

Conflicts of interest E.A.B.: Research grant-in-aid from Boston Scientific.

R.N.B.: None to declare.

\section{References}

1. Matsuo K, Fullerton ME, Moeini A (2016) Treatment patterns and survival outcomes in patients with cervical cancer complicated by complete uterine prolapse: a systematic review of literature. Int Urogynecol J 27:29-38

2. Green JA, Kirwan JM, Tierney JF, Symonds P, Fresco L, Collingwood M, Williams CJ (2001) Survival and recurrence after concomitant chemotherapy and radiotherapy for cancer of the uterine cervix: a systematic review and meta-analysis. Lancet 358:781-786 